Pacific Journal of Mathematics

APPROXIMATION THEOREMS FOR MARKOV OPERATORS 


\section{APPROXIMATION THEOREMS FOR MARKOV OPERATORS}

JAMES R. BROWN

Let $(X, \mathscr{F}, m)$ be a totally $\sigma$-finite measure space. A Markov operator (with invariant measure $m$ ) is a positive operator $T$ on $L_{\infty}(X, \mathscr{F}, m)$ such that $T 1=1$ and $\int T f d m=$ $\int f d m$ for all $f \in L_{1}(X, \mathscr{F}, m) \cap L_{\infty}(X, \mathscr{F}, m)$. If $\varphi$ is an invertible measure-preserving transformation of $(X, \mathscr{F}, m)$, then $\varphi$ determines a Markov operator $T_{\varphi}$ by the formula $T_{\varphi} f(x)=$ $f(\varphi x)$. The set $M$ of all Markov operators is convex and each $T_{\varphi}$ is an extreme point.

In case $(X, \mathscr{F}, m)$ is a finite, homogeneous, nonatomic measure space, $M$ may be identified with the set of all doubly stochastic measures on the product space $(X \times X, \mathscr{F} \times \mathscr{F}$, $m \times m)$. The main result of the present paper is that $M$ is compact in the weak operator topology of operators on $L_{2}(X, \mathscr{F}, m)$ and that the set $\Phi$ of operators $T_{\varphi}$ is dense in $M$. It follows that $M$ is the closed convex hull of $\Phi$ in the strong operator topology. We shall further show that $\Phi$ is closed in the uniform operator topology and that the closure of $\Phi$ in the strong operator topology is the set $\Phi_{1}$ of all (not necessarily invertible) measure-preserving transformations of $(X, \mathscr{F}, m)$.

We shall denote $L_{p}(X, \mathscr{F}, m), 1 \leqq p \leqq \infty$, more briefly by $L_{p}$. The operators $T_{\varphi}$ arising from invertible measure-preserving transformations are in certain respects the most pleasant of all Markov operators (in particular, they are unitary). Therefore, it seems worthwhile to determine what role they play in the structure of $M$.

Interest in special cases of this problem is indicated by the attention which has been devoted to the solution of Birkhoff's Problem 111, which is concerned with the case of a denumerable space $X$ with a measure $m$ uniformly distributed on the points of $X$. Thus J. R. Isbell [4], B. A. Rattray and J. E. L. Peck [12] and D. G. Kendall [6] have given approximation theorems for doubly stochastic matrices in terms of convex combinations of permutation matrices. A second type of solution has been given by Kendall [6] and by Isbell [5]. They have shown that the set of permutation matrices coincides with the set of extreme points of $M$. Still a third type of solution has been offered by P. Révész [13], who has shown (essentially) that every

Received September 21, 1964. This paper contains a portion of the author's dissertation presented for the degree of Doctor of Philosophy at Yale University. 
doubly stochastic matrix is an integral over the set of permutation matrices.

In the case of the real line $X$ with Lebesgue measure $m$, Peck [11] has given a solution of the first type above for the set $M$ of what he calls doubly stochastic measures. He also alludes to the corresponding result for the unit interval with Lebesgue measure. In $\S 3$ we shall show that, for finite measure spaces, the set of doubly stochastic measures can be identified with the set of Markov operators. This is not the case for a nonfinite measure space. However, approximation theorems for Markov operators always imply the corresponding approximation theorems for doubly stochastic measures or matrices.

In this paper we shall restrict our attention to finite measure spaces. The $\sigma$-finite case is more complicated and will be considered at a later time.

In $\S 4$ we give a solution of the above-mentioned problem (Theorem 1) for the case of a nonatomic, finite measure space. This is an approximation theorem like that of Peck [11], but is a stronger result in that the convex closure of the set $\Phi$ of invertible measure-preserving transformations of $(X, \mathscr{F}, m)$ is replaced by the closure of $\Phi$. The topology on $M$ is the weak operator topology for operators on $L_{2}$. Simple examples can be constructed to show that the second and third types of solutions mentioned above for doubly stochastic matrices do not extend to doubly stochastic measures on a nonatomic measure space. The Choquet representation theorem can be invoked to give solutions of the third type as integrals over the set of extreme points of $M$. These extreme points have recently been characterized by J. Lindenstrauss [7], but this approach will not be pursued here.

In $\S 5$ we consider the closure and the convex closure of $\Phi$ in the strong operator topology and the uniform operator topology. The results of that section are obtained directly from Theorem 1 and an interesting geometric characterization (Theorem 5) of the operators $T_{\varphi}$ arising from measure-preserving transformations.

The author would like to express his gratitude to Prof. S. Kakutani and Prof. R. M. Blumenthal for valuable discussions of the problem considered in this paper. In particular, acknowledgement is made of certain observations of Prof. Blumenthal which led to considerable simplification in the proof of Theorem 1.

2. Markov operators, A Markov process with discrete time parameter and state space $(X, \mathscr{F})$ is determined (cf. [1], p. 190) by a stochastic transition function $P(x, B)$, i.e. a nonnegative function of $x \in X, B \in \mathscr{F}$ such that

(i) $P(x, B)$ is a probability measure in $B$ for each fixed $x \in X$; 
(ii) $P(x, B)$ is a measurable function of $x$ for each fixed $B \in \mathscr{F}$. We assume, in addition, that $m$ is an invariant measure for $P(x, B)$ in the sense that

(iii)

$$
\int_{X} P(x, B) m(d x)=m(B), \quad B \in \mathscr{F} .
$$

Under these conditions $P(x, B)$ defines a bounded linear operator $T$ on $L_{\infty}$ by the formula

$$
T f(x)=\int_{x} f(y) P(x, d y) .
$$

The operator $T$ clearly has the properties:

$$
\begin{gathered}
f \geqq 0 \Longrightarrow T f \geqq 0 \\
T 1=1 \\
\int_{X} T f(x) m(d x)=\int_{X} f(x) m(d x), \quad f \in L_{\infty} .
\end{gathered}
$$

For instance, equation (5) follows from condition (iii) when $f$ is a characteristic function of a measurable set and more generally by an approximation argument. Likewise, (4) follows from (i). We shall refer to any linear operator $T$ on $L_{\infty}$ which satisfies (3), (4) and (5) as a Markov operator with invariant measure $m$, or simply a Markov operator.

It follows from (3) and (4) that $T$ is a positive operator on $L_{\infty}$ with $\|T\|_{\infty}=1$ and from (5) that $T$ may be uniquely extended to a positive operator on $L_{1}$ with $\|T\|_{1}=1$. This extension is again given by (2). According to the Riesz convexity theorem, $T$ is a contraction operator on $L_{p}$ for each $p, 1 \leqq p \leqq \infty$. That is, $T$ is a positive operator with $\|T\|_{p} \leqq 1$.

The adjoint $T^{*}$ of $T$ defined by

$$
\left(T^{*} f, g\right)=(f, T g)=\int_{x} f(x) T g(x) m(d x)
$$

for $f \in L_{p}, g \in L_{q}, \quad 1 \leqq p, q \leqq \infty, 1 / p+1 / q=1$, is also a Markov operator. Indeed, equation (5) is equivalent to

$$
T * 1=1 \text {. }
$$

Thus equations (3), (4) and $\left(5^{*}\right)$ may be taken as the definition of a Markov operator.

In general, a Markov operator can not be defined in terms of a stochastic transition function. However, under suitable separability restrictions on $(X, \mathscr{F}, m)$ there will always exist a stochastic transition 
function $P(x, B)$ such that (2) holds almost everywhere for each $f \in L_{\infty}$ (cf. [1], pp. $29 \mathrm{ff}$ ).

A measurable transformation (function, mapping) $\varphi$ from $(X, \mathscr{F})$ into itself is said to be measure-preserving if

$$
m\left(\varphi^{-1} B\right)=m(B), \quad B \in \mathscr{F} .
$$

It follows that $\varphi$ is essentially onto, i.e. the complement of its range has measure zero. As usual we shall identify functions which are equal almost everywhere. Hence we may assume that $\varphi$ is onto. If it is also one-to-one and if $\varphi^{-1}$ is measurable, we shall say that $\varphi$ is an invertible measure-preserving transformation. It follows that $\phi^{-1}$ is also measure-preserving.

The correct notion for our purposes is actually that of a measurepreserving set function from $\mathscr{F}$ into $\mathscr{F}$. However, for the measure spaces that we shall be considering in this paper, namely, products of the unit interval with the product Lebesgue measure, every measurepreserving set transformation $\psi$ is given by a measure-preserving point transformation $\varphi, \psi(B)=\varphi^{-1}(B)$.

Let us denote by $\Phi$ the set of all invertible measure-preserving transformations of $(X, \mathscr{F}, m)$ (or rather all equivalence classes of such transformations modulo null transformations) and by $M$ the set of all Markov operators. Then $\Phi$ may be identified with a subset of $M$ by the correspondence $\varphi \rightarrow T_{\varphi}$ where

$$
T_{\varphi} f(x)=f(\varphi x), \quad f \in L_{p} .
$$

We shall show in $\S 4$ that, subject to a homogeneity condition on $(X, \mathscr{F}, m), \Phi$ is dense in $M$ in the weak operator topology.

3. Doubly stochastic measures. It is well known that any finite measure space $(X, \mathscr{F}, m)$ with $m(X)=1$ which is nonatomic and for which there exists a countable class $\mathscr{F}_{0}$ of measurable sets that generates $\mathscr{F}$ is measure-theoretically equivalent to the unit interval. That is, there exists a one-to-one mapping (modulo sets of measure zero) of $\mathscr{F}$ onto the class of Borel subsets of the unit interval which preserves set operations and the measure ([3], p. 173).

More generally, if $m$ is any finite measure, it can be shown [8] that $X$ is essentially a countable union of measurable sets $X_{n}$ which are measure-theoretically equivalent either to a single point or to a product of intervals with the product Lebesgue measure. The measure spaces $\left(X_{n}, \mathscr{F} \cap X_{n}, m\right)$ are homogeneous in the sense that

(i) there exists a class $\mathscr{F}_{n}$ of measurable subsets of $X_{n}$ which generates $\mathscr{F} \cap X_{n}$ and

(ii) for each subset $Y$ of $X_{n}$ of positive measure, the $\sigma$-algebra 
$\mathscr{F} \cap Y$ is not generated by any class of smaller cardinality than that of $\mathscr{F}_{n}$. The cardinality of $\mathscr{F}_{n}$ is called the character of $X_{n}$ and determines the number of copies of the unit interval which go into its representation as a product measure space. It is clear that, except for the atoms (character 1), each of the spaces $X_{n}$ may be assumed to be of different character, hence not measure-theoretically equivalent to each other. In particular, any invertible measure-preserving transformation of $X$ must leave invariant each of the nonatomic $X_{n}$ as well as their union.

Now suppose that $X=X_{1} \cup X_{2}$ where $X_{1}$ and $X_{2}$ are disjoint and $X_{1}$ is either one of the nonatomic $X_{n}$ of the preceding paragraph or the union of all of the atoms. Consider the Markov operator $T$ defined on $L_{p}$ by

$$
T f(x)=\int_{X} f(y) m(d y)
$$

For this operator we have

$$
\left(\chi_{x_{1}}, T \chi_{x_{2}}\right)=\int_{X} \chi_{X_{1}}(x) T \chi_{x_{2}}(x) m(d x)=m\left(X_{1}\right) m\left(X_{2}\right)
$$

(Here and in the remainder of the paper $\chi_{A}$ denotes the characteristic function of the set $A_{\text {。 }}$ ) On the other hand, if $\varphi$ is any measure preserving transformation of $X$, then we know that

$$
\left(\chi_{x_{1}}, T_{\varphi} \chi_{x_{2}}\right)=m\left(X_{1} \cap \varphi^{-1} X_{2}\right)=0 \text {. }
$$

Thus $T$ cannot be a limit of convex combinations of elements of $\Phi$ in any operator topology. We therefore assume that $(X, \mathscr{F}, m)$ is nonatomic and homogeneous. As noted above, this implies that $(X, \mathscr{F}, m)$ is essentially a product of unit intervals with the product Lebesgue measure. As such it has a natural topology, the product topology, in which $X$ is compact, $\mathscr{F}$ coincides with the class of Borel sets of $X$ and $m$ is a regular Borel measure.

Now let $T$ be a Markov operator on $L_{\infty}$. We shall denote the product measure space $(X \times X, \mathscr{F} \times \mathscr{F})$ by $\left(X^{2}, \mathscr{F}^{2}\right)$. We shall further denote the algebra of finite unions of measurable rectangles $A \times B, A, B \in \mathscr{F}$, by $\mathscr{F}_{0}{ }^{2}$. For each such rectangle we define

$$
\lambda(A \times B)=\left(\chi_{A}, T \chi_{B}\right) .
$$

Since $\lambda$ is additive in $A$ and $B$ individually, it follows that it can be uniquely extended, by additivity, to a finitely additive, nonnegative set function on $\mathscr{F}_{0}^{2}$. Moreover,

$$
\lambda(A \times X)=\lambda(X \times A)=m(A), \quad A \in \mathscr{F} .
$$


We shall show that $\lambda$ is countably additive. This is a special case of a theorem of E. Marczewski and C. Ryll-Nardzewski $[9,10]$ on nondirect products of compact measures. For completeness and because the special case is much simpler than the general case, we include a proof.

Any nonnegative, finitely additive set function $\lambda$ on $\mathscr{F}_{0}^{2}$ which satisfies (11) will be said to be doubly stochastic.

LEMma. Let $\lambda$ be a doubly stochastic set function on $\mathscr{F}_{0}^{2}$. Then $\lambda$ is countably additive and regular, hence it has a unique extension to a doubly stochastic measure on $\mathscr{F}^{2}$.

Proof. If $A$ and $B$ are Borel subsets of $X$, then by the regularity of $m$ there exist compact sets $A_{1}$ and $B_{1}$ such that $A_{1} \subset A, B_{1} \subset B$, $m\left(A-A_{1}\right)<\varepsilon$ and $m\left(B-B_{1}\right)<\varepsilon$, where $\varepsilon$ is any preassigned positive number. It follows that $A_{1} \times B_{1} \subset A \times B, A_{1} \times B_{1}$ is compact, and

$$
\begin{aligned}
\lambda\left(A \times B-A_{1} \times B_{1}\right) & \leqq \lambda\left(A \times\left(B-B_{1}\right)\right)+\lambda\left(\left(A-A_{1}\right) \times B\right) \\
& \leqq m\left(B-B_{1}\right)+m\left(A-A_{1}\right)<2 \varepsilon .
\end{aligned}
$$

Thus any set in $\mathscr{F}_{0}{ }^{2}$ can be approximated from the inside by compact sets, i.e. $\lambda$ is regular. It follows by Alexandroff's theorem ([2], p. 138) that $\lambda$ is countably additive. The existence and uniqueness of the extension then follow from the Hahn extension theorem.

COROLLARY. The relation (10) determines a one-to-one correspondence between the set $M$ of Markov operators on $L_{\infty}$ and the set of all doubly stochastic measures on $\mathscr{F}^{2}$.

Proof. We have shown that each Markov operator $T$ determines a unique doubly stochastic measure $\lambda$ satisfying (10). Conversely, suppose that $\lambda$ is a doubly stochastic measure. Let $g \in L_{\infty}$ and let $f$ be a simple function on $(X, \mathscr{F}, m)$. Set

$$
G(f)=\int f(x) g(y) \lambda(d x, d y) \text {. }
$$

Then

$$
\begin{aligned}
|G(f)| & \leqq\|g\|_{\infty} \int|f(x)| \lambda(d x, d y) \\
& =\|g\|_{\infty}\|f\|_{1} .
\end{aligned}
$$

Thus (12) defines for each $g \in L_{\infty}$ a bounded linear functional $G$ on a dense subset of $L_{1}$. It follows that there exists a function $T g \in L_{\infty}$ such that 


$$
(f, T g)=\int f(x) g(y) \lambda(d x, d y)
$$

for all $f \in L_{1}$ 。 If $g \geqq 0$, then $G$ is a positive functional so that $T g \geqq 0$. Thus $T$ is a positive linear operator on $L_{\infty}$. Clearly, $T 1=1$. Moreover,

$$
\int T g(x) m(d x)=\int g(y) \lambda(d x, d y) \text {. }
$$

Since $\lambda$ is doubly stochastic, it follows that $\int T g=\int g$ for all simple functions $g$ and hence for all $g \in L_{\infty}$. Thus $T$ is a Markov operator. Since (10) is clearly a special case of (13), the proof is complete.

Suppose that $T=T_{\varphi}$ is determined by a measure-preserving transformation $\varphi \in \Phi$. Then according to (10) and (9) $\varphi$ determines the doubly stochastic measure $\lambda_{\varphi}$ defined by

$$
\lambda_{\varphi}(A \times B)=m\left(A \cap \varphi^{-1} B\right) .
$$

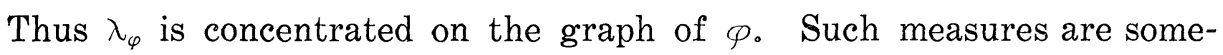
times referred to as permutation measures.

4. Weak approximation. We are now in a position to prove the main result of this paper.

THEOREM 1. $M$ is a compact convex set of operators and $\Phi$ is dense in $M$ in the weak operator topology of $L_{p}, 1<p<\infty$. If $(X, \mathscr{F}, m)$ is a separable measure space, then $M$ is metrizable.

Proof. The convexity of $M$ is clear. Let us show that $M$ is compact. Suppose that $T$ belongs to the closure of $M$ in the weak operator topology of bounded linear operators on $L_{p}$. Since $L_{\infty} \subset L_{p} \cap L_{q}$, where $1 / p+1 / q=1$, and since $(f, T g)$ is a continuous function of $T$ for each fixed $f \in L_{p}, g \in L_{q}$, it follows that $T$ has the properties (3)(5) of $\S 2$. For instance, (3) is equivalent to

$$
f \geqq 0, g \geqq 0 \Rightarrow(f, T g) \geqq 0 .
$$

It follows from (3) and (4) that $T$ maps $L_{\infty}$ into itself. Thus $T$ is a Markov operator and $M$ is closed. Since $L_{p}$ is reflexive, the closed unit sphere in the space of bounded linear operators on $L_{p}$ is compact ([2], p. 512). Since $\|T\|_{p} \leqq 1$ for each $T \in M$, it follows that $M$ is compact.

Note that each of the weak operator topologies corresponding to different values of $p$ is stronger than the weak topology on $M$ determined by the functionals $(f, T g)$ for $f, g \in L_{\infty}$. Since the latter is, nevertheless, a Hausdorff topology and since $M$ is compact in each of 
the weak operator topologies, it follows that they all coincide and hence there is no ambiguity in referring to the weak operator topology on $M$.

Now suppose that $(X, \mathscr{F}, m)$ is separable. Then $L_{p}$ and $L_{q}$ are separable in their norm topologies. Let $\left\{f_{n}\right\}$ and $\left\{g_{n}\right\}$ be dense sequences in $L_{p}$ and $L_{q}$, respectively. The series

$$
\rho(S, T)=\sum_{n=1}^{\infty} \sum_{m=1}^{\infty} \frac{1}{2^{n \leftarrow m}} \cdot \frac{\left|\left(f_{n},(T-S) g_{m}\right)\right|}{1+\left|\left(f_{n},(T-S) g_{m}\right)\right|}
$$

is uniformly convergent in $S$ and $T$ and thus defines a continuous (weak operator topology) metric on $M$. It follows that the resulting metric topology is weaker than the weak operator topology and hence, by the compactness of $M$, that the two topologies coincide.

It only remains to show that $\Phi$ is dense in $M$. A basis for the weak operator topology of $M$ is given by all sets of the form

$$
\left\{T:\left\{\left(f_{k}, T g_{k}\right)-\left(f_{k}, T_{0} g_{k}\right) \mid<\varepsilon, k=1, \cdots, n\right\}\right.
$$

where $f_{k}$ and $g_{k}$ run through a dense subset of $L_{2}, T_{0} \in M$ and $\varepsilon>0$. In particular, we may take $f_{k}$ and $g_{k}$ to be continuous. (Recall that $X$ has a natural topology for which $X$ is compact and $m$ is regular.) In this case they are bounded and, by the arbitrariness of $\varepsilon$ in (15), we may assume that they are bounded by 1 . Let $T_{0} \in M$. We shall show that there exists a measure-preserving transformation $\varphi$ such that $T_{\varphi}$ belongs to the set (15).

For $A, B \in \mathscr{F}$ we introduce the notation

$$
\begin{aligned}
\lambda_{0}(A \times B) & =\left(\chi_{A}, T_{0} \chi_{B}\right) \\
\lambda_{\varphi}(A \times B) & =\left(\chi_{A}, T_{\varphi} \chi_{B}\right)=m\left(A \cap \varphi^{-1} B\right), \quad \varphi \in \Phi .
\end{aligned}
$$

According to the lemma of $\S 3, \lambda_{0}$ and $\lambda_{\varphi}$ may be extended to doubly stochastic measures on $\left(X^{2}, \mathscr{F}^{2}\right)$. Let us set $h_{k}(x, y)=f_{k}(x) g_{k}(y)$, $k=1, \cdots, n$. According to (13), we have

$$
\left(f_{k}, T_{0} g_{k}\right)=\int_{X^{2}} h_{k} d \lambda_{0}
$$

and

$$
\left(f_{k}, T_{\varphi} g_{k}\right)=\int_{X^{2}} h_{k} d \lambda_{\varphi}
$$

Since each $h_{k}$ is uniformly continuous on $X^{2}$, it follows that there exist disjoint sets $X_{1}, \cdots, X_{s} \in \mathscr{F}$ such that $X=\bigcup_{i=1}^{s} X_{i}$ and the oscillation of each $h_{k}$ is less than $\varepsilon / 3$ on each rectangle $X_{i} \times X_{j}$, $i, j=1, \cdots, s$. Since $\lambda_{0}$ is doubly stochastic, we can choose, for each $i=1, \cdots, s$, disjoint measurable subsets $X_{i j}$ of $X_{i}$ such that $m\left(X_{i j}\right)=$ 
$\lambda_{0}\left(X_{i} \times X_{j}\right), j=1, \cdots, s_{\circ}$ (Recall that $X$ is nonatomic.) Similarly, for each $j=1, \cdots, s$ we can choose disjoint measurable subsets $Y_{i j}$ of $X_{j}$ such that $m\left(Y_{i j}\right)=\lambda_{0}\left(X_{i} \times X_{j}\right), i=1, \cdots, s$. For each $i$ and $j$ there exists an invertible measure-preserving transformation $\varphi_{i j}$ of $X_{i j}$ onto $Y_{i j}$ since $X_{i j}$ and $Y_{i j}$ are both homogeneous with the same character and the same measure. Define $\varphi$ on $X$ by

$$
\varphi(x)=\varphi_{i j}(x), \quad x \in X_{i j} .
$$

We shall show that $T_{\varphi}$ belongs to the set (15)。

Since $\varphi$ maps each $X_{i r}$ onto $Y_{i r} \subset X_{r}$ and since $X_{i}=\dot{\cup}_{r=1}^{s} X_{i r}$, it follows that

$$
\lambda_{\varphi}\left(X_{i} \times X_{j}\right)=m\left(X_{i} \cap \varphi^{-1} X_{\jmath}\right)=m\left(X_{i j}\right)=\lambda_{0}\left(X_{i} \times X_{j}\right)
$$

for each $i, j=1, \cdots, s$. Recalling that the oscillation of each of the functions $h_{k}$ is less than $\varepsilon / 3$ on each of the sets $X_{i} \times X_{j}$ and that $\left|h_{k}(x, y)\right| \leqq 1$, we have

$$
\begin{aligned}
\left|\left(f_{k}, T_{\varphi} g_{k}\right)-\left(f_{k}, T_{0} g_{k}\right)\right|= & \left|\int_{X^{2}} h_{k} d \lambda_{\varphi}-\int_{X^{2}} h_{k} d \lambda_{0}\right| \\
\leqq & \sum_{i, j=1}^{s}\left|\int_{X_{i} \times X_{j}} h_{k} d \lambda_{\varphi}-\int_{X_{i} \times X_{j}} h_{k} d \lambda_{0}\right| \\
\leqq & \sum_{i, j=1}^{s}\left\{\varepsilon / 3\left[\lambda_{\varphi}\left(X_{i} \times X_{j}\right)+\lambda_{0}\left(X_{i} \times X_{j}\right)\right]\right. \\
& \left.+\left|\lambda_{\varphi}\left(X_{i} \times X_{j}\right)-\lambda_{0}\left(X_{i} \times X_{j}\right)\right|\right\} \\
= & 2 \varepsilon / 3 \sum_{i, j=1}^{s} \lambda_{v}\left(X_{i} \times X_{\jmath}\right)<\varepsilon
\end{aligned}
$$

and the proof of Theorem 1 is complete.

5. Strong and uniform approximation. Since convex sets have the same closure in the weak operator and the strong operator topologies ([2], p. 447), we immediately obtain the following approximation theorem.

TheOREM 2. $M$ is the closed convex hull of $\Phi$ in the strong operator topology.

It is natural to ask whether Theorems 1 and 2 can be strengthened to give $M$ as the closure of $\Phi$ in the strong operator topology. The answer, at least on $L_{2}$, is negative as we shall now show.

Henceforth, all operator topologies will refer to operators on $L_{2}$ 。

Let us denote by $\Phi_{1}$ the set of all (not necessarily invertible) measure-preserving transformations of $(X, \mathscr{F}, m)$. Again we identify $\Phi_{1}$ with a subset of $M$ by setting $T_{\varphi} f(x)=f(\varphi x), f \in L_{2}$. It follows that $\Phi \subset \Phi_{1} \subset M$. 
THEOREM 3. $\Phi_{1}$ is the closure of $\Phi$ in the strong operator topology.

THEOREM 4. $\Phi$ is closed in the uniform operator topology.

Theorems 3 and 4 follow easily from Theorem 2 and a pair of simple algebraic propositions which we give as Theorem 5 below. Note that nothing is said about the closed convex hull of $\Phi$ in the uniform operator topology. This is apparently still an open question.

THEOREM 5. Let $T$ be a bounded linear operator on $L_{2}$. Then $T \in \Phi_{1}$ if and only if $T$ is doubly stochastic and isometruc; $T \in \Phi$ if and only if $T$ is doubly stochastic and unitary.

Proof. It is well known that every $T \in \Phi_{1}$ is isometric while every $T \in \Phi$ is unitary. Moreover, $T$ is unitary if and only if both $T$ and $T^{*}$ are isometric. Thus the second part of the theorem follows from the first. It only remains to show that every $T \in M$ which is isometric is induced by a measure-preserving transformation $\varphi$ of $X$.

Suppose that $T \in M$ is isometric and let $A$ and $B$ be measurable subsets of $X$. Then

$$
\begin{aligned}
\int_{X}\left(T \chi_{A}\right)\left(T \chi_{B}\right) d m & =\left(T \chi_{A}, T \chi_{B}\right)=\left(\chi_{A}, \chi_{B}\right) \\
& =\left(\chi_{A \cap B}, 1\right)=\left(T \chi_{A \cap B}, 1\right) \\
& =\int_{X} T \chi_{A \cap B} d m
\end{aligned}
$$

However, since $T$ is positive and $0 \leqq \chi_{A} \leqq 1$, we have that $0 \leqq T \chi_{A} \leqq$ $T 1=1$ and so

$$
0 \leqq\left(T \chi_{A}\right)^{2} \leqq T \chi_{A} \leqq 1
$$

It follows from (17) and (16) with $A=B$ that $\left(T \chi_{A}\right)^{2}=T \chi_{A}$ and hence that $T \chi_{A}$ is (essentially) the characteristic function of some measurable set. Let us denote this set by $\psi(A)$.

Using the positivity of $T$ again we have, moreover, that $T \chi_{A \cap B} \leqq$ $\min \left\{T \chi_{A}, T \chi_{B}\right\}$ so that

$$
0 \leqq T \chi_{A \cap B}=\left(T \chi_{A \cap B}\right)^{2} \leqq\left(T \chi_{A}\right)\left(T \chi_{B}\right) .
$$

From (16) and (18) we see that

$$
T \chi_{A \cap B}=\left(T \chi_{A}\right)\left(T \chi_{B}\right)
$$

or

$$
\psi(A \cap B)=\psi(A) \cap \psi(B)
$$


for all measurable sets $A$ and $B$. Thus $\psi$ preserves finite intersections. From the relations $\chi_{A \cup B}=\chi_{A}+\chi_{B}-\chi_{A \cap B}$ and $\chi_{A^{\prime}}=1-\chi_{A}$ and the fact that $T 1=1$, it follows that $\psi$ preserves finite unions and complements as well. From the relation

$$
m(\psi(A))=\left(T \chi_{A}, T 1\right)=\left(\chi_{A}, 1\right)=m(A)
$$

it follows that $\psi$ preserves countable unions and intersections as well as the measure.

It follows that there exists a measure-preserving transformation $\varphi$ of $(X, \mathscr{F}, m)$ such that $\psi(A)=\varphi^{-1}(A)$ for all $A \in \mathscr{F}$. Thus $T \chi_{A}(x)=$ $\chi_{\psi(A)}(x)=\chi_{A}(\varphi x)$. It follows that $T$ coincides with $T_{\varphi}$ on all simple functions and hence on all of $L_{2}$. This completes the proof of Theorem 5 .

It follows immediately from Theorem 5 that $\Phi$ is closed in the uniform operator topology and that $\Phi_{1}$ is closed in the strong operator topology. According to Theorem 2, every element $T \in \Phi_{1}$ is the limit in the weak operator topology of a convergent net $T_{\alpha}$ of elements of $\Phi$. It follows that

$$
\left\|\left(T-T_{\alpha}\right) f\right\|_{2}^{2}=2(f, f)-\left(T f, T_{\alpha} f\right)-\left(T_{\alpha} f, T f\right) \rightarrow 0
$$

for each $f \in L_{2}$. Thus $T_{\alpha} \rightarrow T$ and $\Phi$ is dense in $\Phi_{1}$ in the strong operator topology. This completes the proofs of Theorems 3 and 4 .

\section{BIBLIOGRAPHY}

1. J. L. Doob, Stochastic Processes, New York, 1953.

2. N. Dunford and J. T. Schwartz, Linear Operators, Part I, New York, 1958.

3. P. R. Halmos, Measure Theory, Princeton, 1950.

4. J. R. Isbell, Birkhoff's problem 111, Proc. Amer. Math. Soc. 6 (1955), 217-218.

5. - Infinite doubly stochastic matrices, Canad. Math. Bull. 5 (1962), 1-4.

6. D. G. Kendall, On infinite doubly stochastic matrices and Birkhoff's problem 111,

J. London Math. Soc. 35 (1960), 81-84.

7. J. Lindenstrauss, $A$ remark on extreme doubly stochastic measures (unpublished).

8. D. Maharam, On homogeneous measure algebras, Proc. Nat. Acad. Sci. USA 28 (1942), 108-111.

9. E. Marczewski, On compact measures, Fund. Math. 40 (1953), 113-124.

10. and C. Ryll-Nardzewski, Remarks on the compactness and non direct products of measures, Fund. Math. 40 (1953), 165-170.

11. J. E. L. Peck, Doubly stochastic measures, Michigan Math. J. 6 (1959), 217-220.

12. B. A. Rattray and J. E. L. Peck, Infinite stochastic matrices, Trans. Roy. Soc. Canada, Sec. III, (3) 49 (1955), 55-57.

13. P. Révész, A probabilistic solution of problem 111 of $G$. Birkhoff, Acta Math. Acad. Scien. Hungaricae 13 (1962), 187-198.

OREgon State University 



\section{PACIFIC JOURNAL OF MATHEMATICS}

EDITORS

H. SAMELSON

Stanford University

Stanford, California

R. M. BLUMENTHAL

University of Washington

Seattle, Washington 98105
*J. DUGUNDJI

University of Southern California

Los Angeles, California 90007

RICHARD ARENS

University of California

Los Angeles, California 90024

\section{ASSOCIATE EDITORS}

E. F. BECKENBACH

B. H. NeumanN

F. WOLF

K. YosidA

\section{SUPPORTING INSTITUTIONS}

UNIVERSITY OF BRITISH COLUMBIA

CALIFORNIA INSTITUTE OF TECHNOLOGY

UNIVERSITY OF CALIFORNIA

MONTANA STATE UNIVERSITY

UNIVERSITY OF NEVADA

NEW MEXICO STATE UNIVERSITY

OREGON STATE UNIVERSITY

UNIVERSITY OF OREGON

OSAKA UNIVERSITY

UNIVERSITY OF SOUTHERN CALIFORNIA
STANFORD UNIVERSITY

UNIVERSITY OF TOKYO

UNIVERSITY OF UTAH

WASHINGTON STATE UNIVERSITY

UNIVERSITY OF WASHINGTON

AMERICAN MATHEMATICAL SOCIETY CHEVRON RESEARCH CORPORATION

TRW SYSTEMS

NAVAL ORDNANCE TEST STATION 


\section{Pacific Journal of Mathematics}

\section{Vol. 16, No. $1 \quad$ November, 1966}

Larry Armijo, Minimization of functions having Lipschitz continuous first

partial derivatives ............................... 1

Edward Martin Bolger and William Leonard Harkness, Some

characterizations of exponential-type distributions.............. 5

James Russell Brown, Approximation theorems for Markov operators ...... 13

Doyle Otis Cutler, Quasi-isomorphism for infinite Abelian p-groups ...... 25

Charles M. Glennie, Some identities valid in special Jordan algebras but not valid in all Jordan algebras .......................... 47

Thomas William Hungerford, A description of $\operatorname{Mult}_{i}\left(A^{1}, \cdots, A^{n}\right)$ by

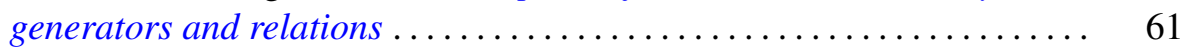

James Henry Jordan, The distribution of cubic and quintic non-residues ....

Junius Colby Kegley, Convexity with respect to Euler-Lagrange differential operators................................... 87

Tilla Weinstein, On the determination of conformal imbedding ......... 113

Paul Jacob Koosis, On the spectral analysis of bounded functions ........ 121

Jean-Pierre Kahane, On the construction of certain bounded continuous functions ................................... 129

V. V. Menon, A theorem on partitions of mass-distribution ........... 133

Ronald C. Mullin, The enumeration of Hamiltonian polygons in triangular

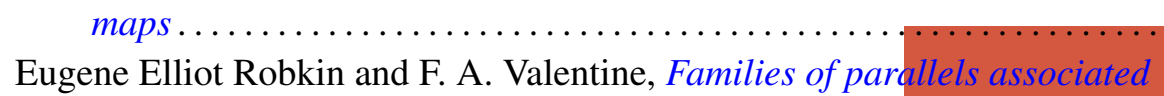

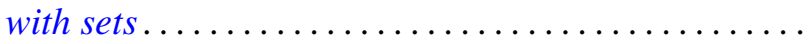

Melvin Rosenfeld, Commutative F-algebras

A. Seidenberg, Derivations and integral closure

S. Verblunsky, On the stability of the set of exponents of a Cauchy

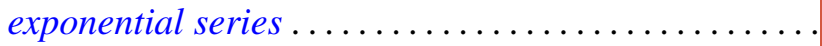

Herbert Walum, Some averages of character sums 